\title{
提高 $\left(\mathrm{K}_{0.5} \mathrm{Na}_{0.5}\right) \mathrm{NbO}_{3}$ 陶瓷压电性能温度稳定性的 新方法
}

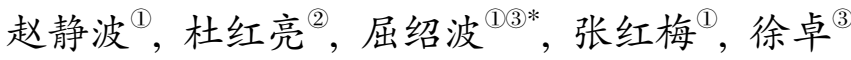 \\ (1) 空军工程大学理学院, 西安 710051; \\ (2) 西安交通大学电力设备电气绝缘国家重点实验室, 西安 710072; \\ (3) 西安交通大学电子材料与器件教育部重点实验室, 西安 710072 \\ *联系人, E-mail: qushaobo@126.com
}

2010-11-26 收稿, 2011-02-21 接受

国家自然科学基金(10804130, 60871027)、陕西省自然科学基金(2009JQ1001)和电力设备电气绝缘国家重点实验室基金(EIPEP10202)资助项目

摘要 分析了 $\left(\mathrm{K}_{0.5} \mathrm{Na}_{0.5}\right) \mathrm{NbO}_{3}$ (简写为 $\mathrm{KNN}$ ) 基陶瓷压电性能温度稳定性差的原因, 结合 $\mathrm{KNN}$ 固 溶体的相图, 提出了解决 $\mathrm{KNN}$ 基陶瓷压电性能温度稳定性差的新方法. 为了验证此方法的可 行性, 设计了 $0.9\left(\mathrm{~K}_{1-x} \mathrm{Na}_{x}\right) \mathrm{NbO}_{3}-0.06 \mathrm{LiNbO}_{3}-0.04 \mathrm{CaTiO}_{3}$ (简记为 $\mathrm{KNLN}^{-\mathrm{CaTiO}}$ ) 陶瓷. 系统研究 了 $\mathrm{KNLN}^{-} \mathrm{CaTiO}_{3}$ 陶瓷的结构和性能. 实验结果表明: $\mathrm{KNLN}-\mathrm{CT}(x=0.54)$ 陶瓷在宽阔的温度范 围 $\left(25 \sim 320^{\circ} \mathrm{C}\right)$ 内表现出良好的温度稳定性, 在 $x=0.54$ 处获得了相对较高的压电性能 $\left(d_{33}=152\right.$

关键词

铌酸钾钠 准同型相界 压电 温度稳定性 $\mathrm{pC} / \mathrm{N})$. 这个结果证实了我们提出的解决 $\mathrm{KNN}$ 基陶瓷压电性能温度稳定性差的方法是可行的, 具有使用价值.

压电陶瓷是一种可实现机械能与电能相互转换 的功能材料, 已被广泛应用于军事、汽车、商业和医 疗技术领域 ${ }^{[1,2]}$. 锆钛酸铅 $\mathrm{Pb}(\mathrm{Zr}, \mathrm{Ti}) \mathrm{O}_{3}(\mathrm{PZT})$ 是目前广 泛应用的压电陶瓷. 由于铅给人类及生态环境带来 严重危害, 世界各国都把高性能无铅压电陶瓷作为 一项迫切的、具有重大社会和经济意义的课题进行研 究和开发 ${ }^{[3 \sim 5]}$.

近年来, 铌酸钾钠 $\left(\mathrm{K}_{0.5} \mathrm{Na}_{0.5}\right) \mathrm{NbO}_{3}$ (简写为 $\mathrm{KNN}$ ) 基无铅压电陶瓷受到了广泛的关注. 因为 $\mathrm{KNN}$ 陶瓷 不仅具有比其他无铅压电陶瓷相对较高的压电性能 $(200 \sim 300 \mathrm{pC} / \mathrm{N})$ 和较高的居里温度 $\left(350 \sim 420^{\circ} \mathrm{C}\right)^{[6 \sim 17]}$, 而且 $\mathrm{KNN}$ 基无铅压电陶瓷与人体组织具有良好的兼 容性, 可以直接植人人的体内, 是对人类和环境非常 友好的材料, 故被认为是非常有前途替代 PZT 的无 铅压电材料 ${ }^{[2]}$. 然而, 目前 KNN 基无铅压电陶瓷遇 到了一个非常严重的问题, 即压电性能具有差的温 度稳定性. Hollenstein 等证实, 当工作温度偏离室温
区域时, $0.94\left(\mathrm{~K}_{0.5} \mathrm{Na}_{0.5}\right) \mathrm{NbO}_{3}-0.06 \mathrm{LiNbO}_{3}$ 陶瓷的压电 性能会降低 $30 \%{ }^{[18]}$. 低的压电性能温度稳定性限制 了 $\mathrm{KNN}$ 基无铅压电陶瓷的实际应用, 因此, 目前关 于 $\mathrm{KNN}$ 基无铅压电陶瓷的研究集中在如何提高压电 性能的稳定性上 ${ }^{[19 \sim 21]}$. 本论文提出一种提高 $\mathrm{KNN}$ 基 陶瓷压电性能温度稳定性的新方法, 并且用实验 验证.

\section{1 研究思路}

研究表明, 造成 KNN 基无铅压电陶瓷压电性能 温度稳定性差的本质原因是在室温附近存在一个从 正交相向四方向转变的多晶相变 ${ }^{[5,10 \sim 15]}$. 根据这个原 因, 本课题组和 Zhang 课题组均提出了通过添加新的 组元, 把这个多晶相变转移到室温以下 ${ }^{[19,22]}$. 研究结 果表明，此方法可以明显提高温度稳定性，但是，也 会同时降低 $\mathrm{KNN}$ 基无铅压电陶瓷的压电性能. 因此, 我们必须寻找新的方法来提高温度稳定性，同时，又

英文版见: Zhao J B, Du H L, Qu S B, et al. Improvement in the piezoelectric temperature stability of $\left(\mathrm{K}_{0.5} \mathrm{Na}_{0.5}\right) \mathrm{NbO}_{3}$ ceramics.Chinese Sci Bull, 2011, 56, doi: $10.1007 / \mathrm{s} 11434-011-4439-6$ 
不会使压电性能大幅度降低.

最近的研究表明, $\mathrm{Na} / \mathrm{K}$ 对 $\mathrm{KNN}$ 基陶瓷的压电性 能有很大的影响 ${ }^{[23,24]}$. 张孝文教授的研究表明, 对于 纯的 $\mathrm{KNN}$ 基陶瓷, 在 $\mathrm{Na} / \mathrm{K}$ 为 $52 / 48$ 时, 存在一个相 界, 这个相界分割开两个不同的正交相, 在 $200^{\circ} \mathrm{C}$ 以 上, 分割开两个不同的四方相, 此相界类似于 PZT 陶 瓷中的准同型相界, 因此, 纯的 $\mathrm{KNN}$ 基陶瓷在 $\mathrm{Na} / \mathrm{K}$ 为 52/48 时, 表现出优异的压电性能 ${ }^{[23]}$. 根据此研究 结果, 我们提出了一个解决 KNN 基陶瓷压电性能的 温度稳定性差, 又不会显著降低压电性能的方法, 即: 通过添加新的组元, 把正交相向四方向转变的多晶 相变点转移到室温以下以提高材料的温度稳定性, 然后通过调整 $\mathrm{Na} / \mathrm{K}$, 寻找分割开两个不同四方相的 相界, 以期在相界处获得高的压电性能.

为了验证此方法的可行性, 设计了如下的实验. 主要设计有 3 个方面考虑: (1) 根据文献[19]报道, $\mathrm{Ca}^{2+}$ 取代不会显著降低居里温度, 但会明显地降低从 正交相向四方向转变的多晶相变温度, 因此我们期 望引人 $\mathrm{CaTiO}_{3}$ 把从正交相向四方向转变的多晶相变 移至室温以下. (2) 由于 $\mathrm{LiNbO}_{3}$ 具有高的居里温度 $\left(\sim 1210^{\circ} \mathrm{C}\right)$, 因此引人 $\mathrm{LiNbO}_{3}$ 提高居里温度. (3) 根据 $\mathrm{KNN}$ 相图, 我们能看到在 $x=0.52$ 附近有一条坚直的 线, 这条线的两侧的铁电相略微不同, 四方相 (FT1 和 FT2) 和正交相(FO1 和 FO2). 因为这条线与 PZT 准 同型相界线类似，所以我们希望能找到这条线对应 的成分点, 从而获取高压电性能和优异的温度稳定 性. 因此通过控制 $\mathrm{Na} / \mathrm{K}$ 来确定相界处最佳组分, 使 得 $\mathrm{KNN}$ 基无铅压电陶瓷在保持良好温度稳定性的同 时保持较高的压电性能. 根据以上分析, 我们设计的
材料配方为: $0.9\left(\mathrm{~K}_{1-x} \mathrm{Na}_{x}\right) \mathrm{NbO}_{3}-0.06 \mathrm{LiNbO}_{3}-0.04 \mathrm{CaTiO}_{3}$, 本论文将对其结构和性能进行系统研究.

\section{2 实验}

采用传统的陶瓷制备工艺技术, 以国药集团化 学试剂有限公司, 中国合肥生产的 $\mathrm{K}_{2} \mathrm{CO}_{3}(\geqslant 99 \%)$, $\mathrm{Na}_{2} \mathrm{CO}_{3}(\geqslant 99.8 \%), \quad \mathrm{Li}_{2} \mathrm{CO}_{3}(\geqslant 98 \%), \quad \mathrm{Nb}_{2} \mathrm{O}_{5}(\geqslant 99.99 \%)$, $\mathrm{CaCO}_{3}(\geqslant 99 \%), \mathrm{TiO}_{2}(\geqslant 99 \%)$ 为起始原料, 根据 $0.9 \mathrm{~K}_{1-x^{-}}$ $\mathrm{Na}_{x} \mathrm{NbO}_{3}-0.06 \mathrm{LiNbO}_{3}-0.04 \mathrm{CaTiO}_{3}$ (简记为 $\mathrm{KNLN}-\mathrm{CaTiO}_{3}$ ) 陶瓷体系的化学计量进行配料, 其中 $x$ 分别为 0.50 , $0.52,0.54,0.56$. 首先将原料混合物振动球磨 $12 \mathrm{~h}$, 充分混合、粉碎后, 然后在 $860^{\circ} \mathrm{C}$, 经 $4 \mathrm{~h}$ 的预烧合 成陶瓷粉体; 合成后的陶瓷粉末充分研磨并过 70 目 分样篮后, 加人适量的黏结剂, 造粒得到流动性好的 颗粒; 在一定压力下干压成型, 获得厚度为 $1.5 \mathrm{~mm}$ 、 直径为 $10 \mathrm{~mm}$ 的生坏片; 并在 $1140^{\circ} \mathrm{C}$ 下烧结 $2 \mathrm{~h}$ 得到 致密的陶瓷片. 将清洗好的陶瓷片用毛刷镀上银电 极 $810^{\circ} \mathrm{C}$ 保温 $2 \mathrm{~h}$. 在硅油温度为室温、极化直流电压 为 $4 \mathrm{kV} / \mathrm{mm}$ 的条件下极化 $30 \mathrm{~min}$, 放置 $24 \mathrm{~h}$ 后, 测 试各项性能. 用 ZJ-3A 准静态测量仪(中国科学院声 学研究所)测量 $d_{33}$; 用 LCR 数字电桥(TH2816A) 在常 温下测得 $1 \mathrm{kHz}$ 时陶瓷样品的介电常数 $\varepsilon_{\mathrm{r}}$ 和介电损耗 $\tan \delta$; 用日本电子 JSM-5800 型扫描电子显微镜观测 陶瓷样品表面和横断面的微观形貌.

\section{3 结果与讨论}

\section{1 相结构和微观结构分析}

图 1(a)是 KNLN-CT 陶瓷的 X 射线衍射图 $\left(25^{\circ} \mathrm{C}\right)$, 图 1(b)为 $45^{\circ}$ 特征峰放大图, 从图 1 中可以看出, 当
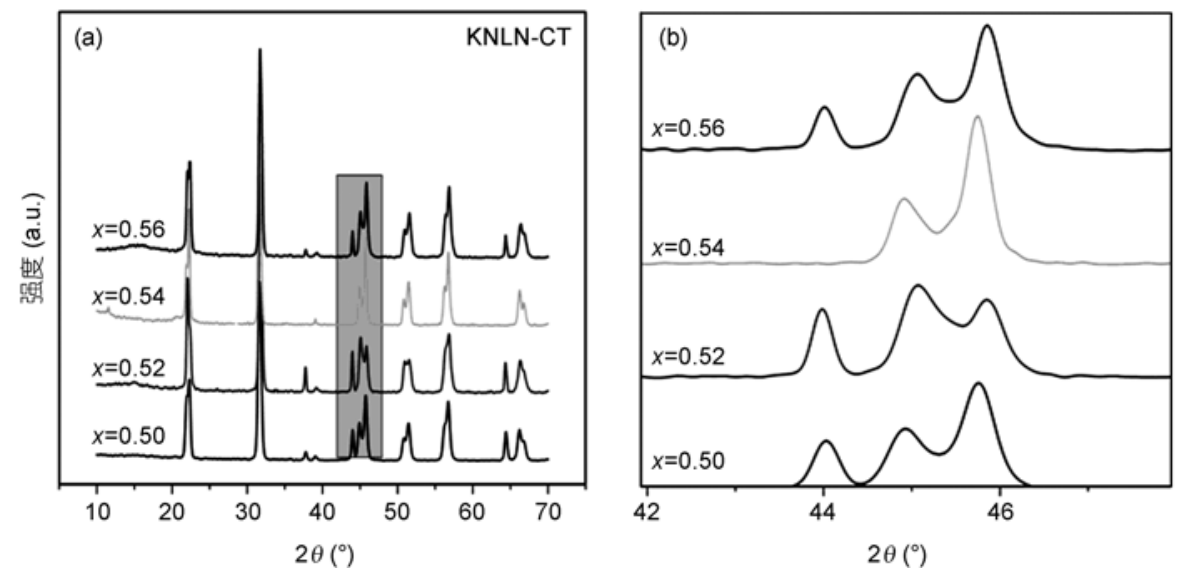

图 1 KNLN-CT 陶瓷 X 射线衍射 
$x=0.50 \sim 0.56$ 时, KNLN-CT 陶瓷均为纯钙钛矿相, 没 有任何杂相生成, 根据我们以前的报道 ${ }^{[22]}$ 和四方相 的特征峰我们可以确定, 当 $x=0.50,0.52,0.56$ 时, KNLN-CT 陶瓷为四方相, 其原因主要是由于 $\mathrm{LiNbO}_{3}$ 和 $\mathrm{CaTiO}_{3}$ 的引人使得正交相向四方相转变的多晶相 变从 $200^{\circ} \mathrm{C}$ 附近转移至室温以下, 因此 KNLN-CT 陶 瓷的相结构为四方相. 另外, 从图 1(b)也可以看出, 当 $x=0.50,0.52,0.56$ 时, $45^{\circ}$ 特征峰均有 3 个峰, 但是 峰的高低和起伏略有不同, 表明晶格常数的略微差 别; 当 $x=0.54$ 时, $45^{\circ}$ 特征峰有两个峰, 过高和过低的 成分都是 3 个峰, 表明 $x=0.54$ 时的相结构可能是两个 不同四方相的混合相. 相结构的结果也可能被下面 的介电温谱证实. 图 2 是 KNLN-CT 陶瓷表面扫描电 子显微镜图从图 2 可以看出, 所有组份的 KNLN-CT 陶瓷样品晶粒尺寸随 $\mathrm{Na} / \mathrm{K}$ 变化不大, 平均尺寸都在
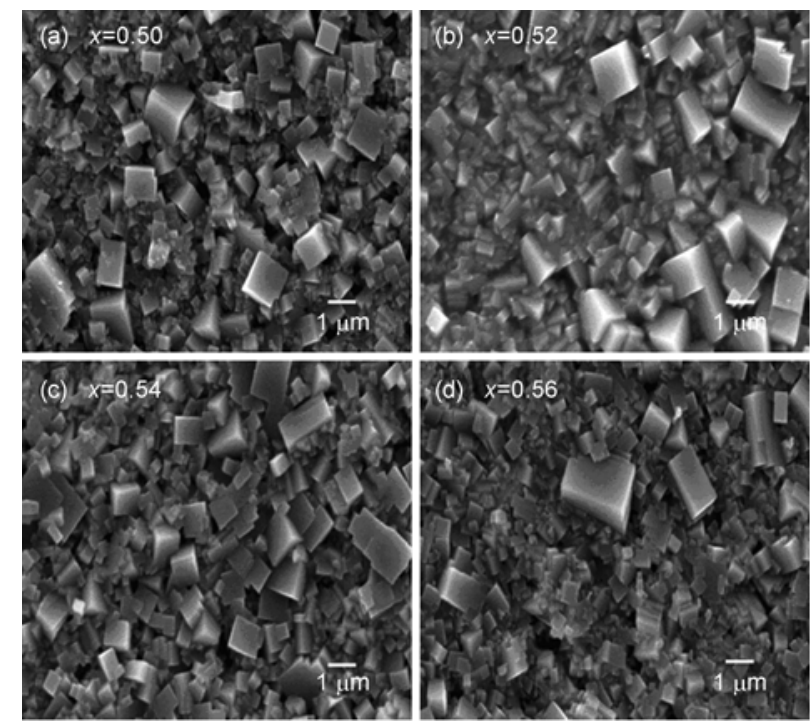

图 2 KNLN-CT 陶瓷表面扫描电子显微镜图
$1 \mu \mathrm{m}$, 晶粒生长良好、晶界清晰、气孔较少, 表明陶 瓷样品比较致密.

\section{2 介电性能分析}

介电温谱通常也是研究铁电材料相结构变化的 一个有力工具, 因为铁电材料在相结构发生变化的 温度区域通常会在介电温谱上出现一个峰值. 图 3(a) 是 KNLN-CT 陶瓷样品的介电温谱 $(100 \mathrm{kHz})$, 可以看 出, 不同 $\mathrm{Na} / \mathrm{K}$ 对 KNLN-CT 陶瓷居里温度的影响不 大, 而且居里温度均在 $360^{\circ} \mathrm{C}$ 附近, 与文献[19]报道 的相比, 居里温度更高, 这主要是由于 $\mathrm{LiNbO}_{3}$ 具有 高的居里温度 $\left(1210^{\circ} \mathrm{C}\right)$, 它的引人有利于 $\mathrm{KNN}$ 基陶 瓷居里温度的提高. 同时从图 3(a)中也能看到, 介电 峰随钠比例的增多先增大后减小, 当 $x=0.54$ 时介电 常数达到最大值 $\varepsilon_{\max }=2323$. 图 3(b) 是正交相向四方 相转变的多晶相变的放大图, 从图中可以看出, 所有 陶瓷样品的正交相向四方相转变的多晶相变温度均 在 $-40^{\circ} \mathrm{C}$ 左右, 表明室温下是四方结构, 这与图 1 分 析结果一致. 正交相向四方相转变的多晶相变温度 移至室温以下, 有利于室温以上压电性能的稳定，大 大降低压电性能对温度的依赖, 这与我们的实验设 计目的一致. 图 3(c) 是不同钾钠比 KNLN-CT 陶瓷 介电损耗图, 从图中可以看出, 在 $25 \sim 350^{\circ} \mathrm{C}$ 范围, 所 有钾钠比 KNLN-CT 陶瓷介电损耗 $\tan \delta<0.05$, 具有 重要的工程应用价值.

\section{3 压电性能分析}

图 4 是 KNLN-CT 陶瓷样品的压电性能, 从图中 可以看出, $\mathrm{Na} / \mathrm{K}$ 对 $\mathrm{KNLN}-\mathrm{CT}$ 陶瓷样品的压电系数有 很大的影响, 随着 $x$ 的增大, 压电常数先增大后降低 且在 $x=0.54$ 时达到最大值 $d_{33}=152 \mathrm{pC} / \mathrm{N}$, 这可能是由
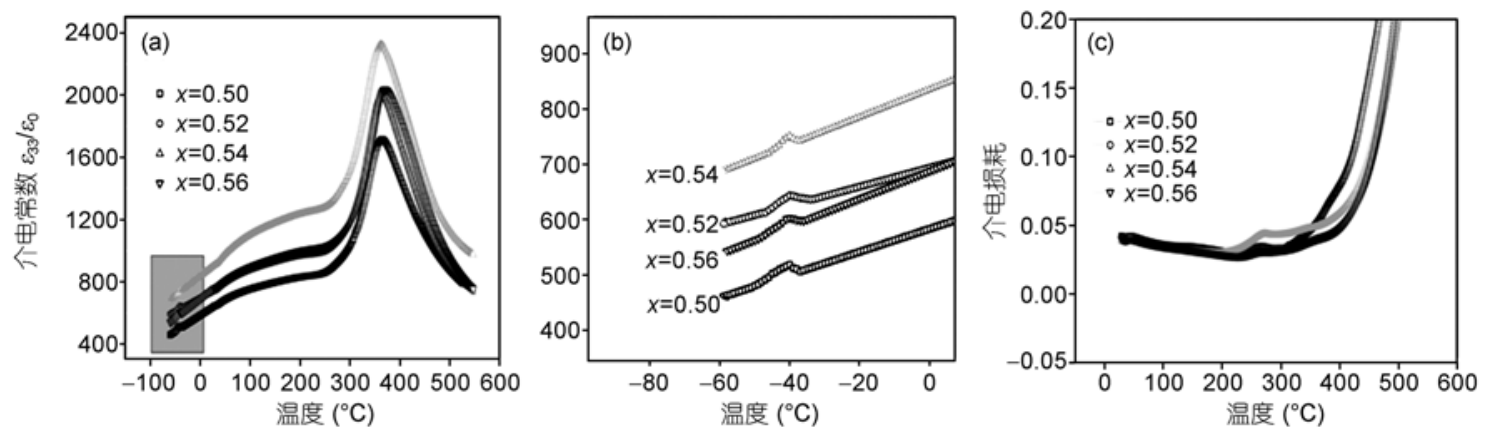

图 3 KNLN-CT 陶瓷不同钾钠比的介电温度性能 


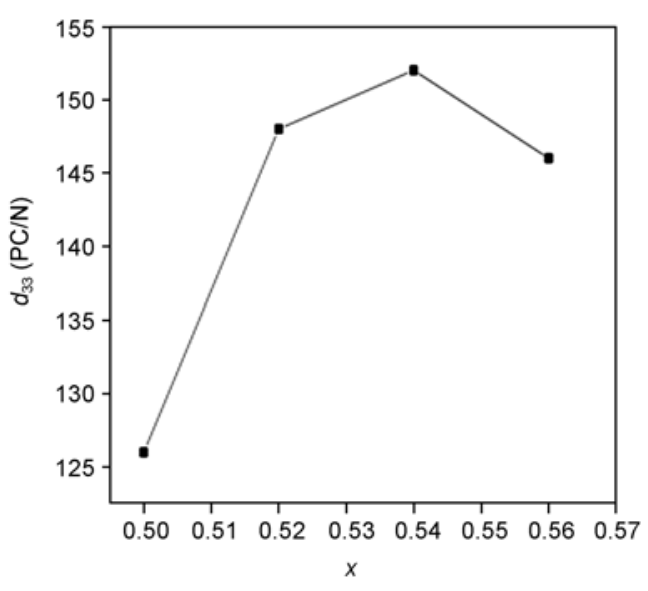

图 4 KNLN-CT 陶瓷不同钾钠比的压电性能

于当 $x=0.54$ 时, KNLN-CT 陶瓷处于两个不同的四方 相共存的准同型相界处, 在相界处, 电畴更容易被定 向, 故压电活性提高; 另一方面, 与目前的研究成果 相比, 压电性能并不是很高, 这主要可以从两个方面 来解释, 首先在 $x=0.54$ 时, KNLN-CT 陶瓷所产生的 准同型相界与 PZT 的准同型相界不同, PZT 的准同型 相界是菱方相和四方相的混合相, 而 KNLN-ST 的准 同型相界是不同四方相的混合相, 这两个相同属四 方相, 只是在晶格常数上略有差别, 这可以从 $\mathrm{X}$ 射线 衍射峰(图 1)分析出, 因此, 极化方向没有增多, 压电 活性虽然有所提高, 但提高有限. 其次, KNLN-CT 陶 瓷的压电性能不高与 $\mathrm{CaTiO}_{3}$ 的引人有关, 由于 $\mathrm{CaTiO}_{3}$ 的引人, $\mathrm{KNN}$ 的 $\mathrm{A}$ 位 $\mathrm{K}^{+}$和 $\mathrm{Na}^{+}$部分被 $\mathrm{Ca}^{2+}$ 取代, 这种 施主取代必定产生 A 空位, 根据 Cochran 的理论 ${ }^{[25]}$, 在 $\mathrm{ABO}_{3}$ 钙钛矿结构中, $\mathrm{A}$ 位离子对于 $\mathrm{BO}_{6}$ 氧八面体 具有巨大的耦合作用, 所以 $\mathrm{A}$ 空位必然减弱这种堣 合作用, 这样就会使得晶胞耦合定向能力减弱, 铁 电畴生长能力不足, 陶瓷的自发极化定向能力降低, 压电性能不高. 此外, 文献[19]报道 $\mathrm{CaTiO}_{3}$ 的引人导 致了 $\mathrm{KNN}$ 陶瓷的弛豫特征, 这也可以解释压电性能 不高的原因. 图 5 是 $\mathrm{KNLN}-\mathrm{CT}(x=0.54)$ 陶瓷在不同 温度下的压电性能, 从图中可以看出, 在 $25 \sim 320^{\circ} \mathrm{C}$ 范围内, 压电系数的变化很小, 这个结果表明 $\operatorname{KNLN}-\mathrm{CT}(x=0.54)$ 陶瓷具有良好的压电性能温度稳 定性.

基于以上的结果我们可以看出, $\mathrm{KNLN}-\mathrm{CT}(x=$ $0.54)$ 陶瓷既具有相对较高的压电系数 $(152 \mathrm{pC} / \mathrm{N})$, 又 表现出良好的温度稳定性. 其原因主要是 $\mathrm{LiNbO}_{3}$ 和

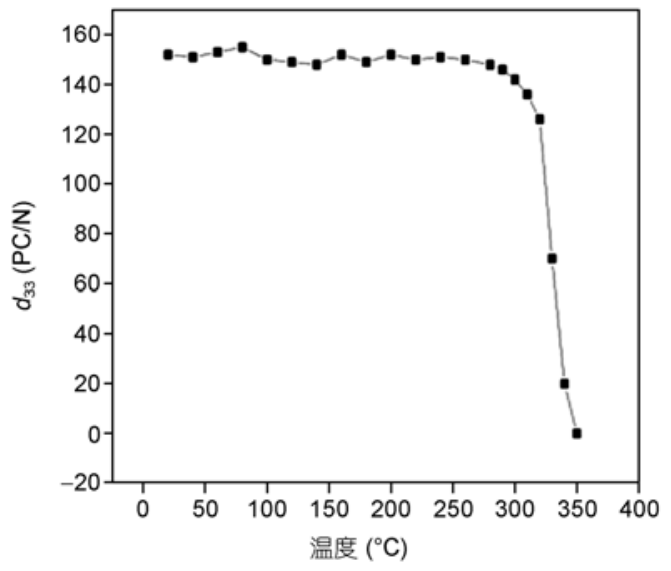

图 5 KNLN-CT 陶瓷 $(x=0.54)$ 不同温度下的压电性能

$\mathrm{CaTiO}_{3}$ 的加人把正交相向四方相转变的多晶相变移 至室温以下 $\left(-40^{\circ} \mathrm{C}\right)$, 故 $\mathrm{KNLN}-\mathrm{CT}(x=0.54)$ 陶瓷表现 出良好的温度稳定性, 当 $x=0.54$ 时, KNLN-CT 陶瓷 出现了类似于准同型相界的相界, 故 KNLN-CT $(x=0.54)$ 陶瓷表现出相对较高的压电性能. 这里必须 指出一点: 尽管 $\mathrm{KNLN}-\mathrm{CT}(x=0.54)$ 陶瓷的压电性能 $\left(d_{33}=152 \mathrm{pC} / \mathrm{N}\right)$ 低于另外的 $\mathrm{KNN}$ 基无铅压电陶瓷的 压电性能, 但是, $\mathrm{KNLN}-\mathrm{CT}(x=0.54)$ 陶瓷具有良好的 温度稳定性, 因此, 相比较而言, $\operatorname{KNLN}-\mathrm{CT}(x=0.54)$ 陶瓷更具有实际应用的价值. 该实验结果也证实了 我们提出的解决 KNN 基陶瓷压电性能温度稳定性差 的方法是可行的，具有使用价值.

\section{4 结论}

采用固相反应烧结法制备了 $0.9\left(\mathrm{~K}_{1-x} \mathrm{Na}_{x}\right) \mathrm{NbO}_{3}-$ $0.06 \mathrm{LiNbO}_{3}-0.04 \mathrm{CaTiO}_{3}$ 陶瓷, 通过 $\mathrm{LiNbO}_{3}$ 和 $\mathrm{CaTiO}_{3}$ 的加人把正交相向四方相转变的多晶相变移至室温 以下 $\left(-40^{\circ} \mathrm{C}\right)$, 使 $\mathrm{KNLN}-\mathrm{CT}(x=0.54)$ 陶瓷在宽阔的温 度范围内表现出良好的温度稳定性, 通过控制 $\mathrm{Na} / \mathrm{K}$ 找到了 KNN 相图中类似于 PZT 中准同型相界的相 界, 在 $x=0.54$ 处获得了相对较高的压电性能 $\left(d_{33}=152\right.$ $\mathrm{pC} / \mathrm{N})$. 这个结果证实了我们提出的解决 $\mathrm{KNN}$ 基陶 瓷压电性能温度稳定性差的方法是可行的, 具有使 用价值. 尽管 KNLN-CT $(x=0.54)$ 陶瓷的压电性能 $\left(d_{33}=152 \mathrm{pC} / \mathrm{N}\right)$ 低于另外的 $\mathrm{KNN}$ 基无铅压电陶瓷的压 电性能 $\left(d_{33}=200 \sim 300 \mathrm{pC} / \mathrm{N}\right)$, 但是, $\operatorname{KNLN}-\mathrm{CT}(x=0.54)$ 陶瓷具有良好的温度稳定性, 因此, 相比较而言, KNLN-CT $(x=0.54)$ 陶瓷更具有实际应用的价值. 


\section{参考文献}

1 Cross E. lead-free at last. Naure, 2004, 432: 24-25

2 Maeder M D, Damjanovic D, Setter N. Lead free piezoelectric materials. J Electroceram, 2004, 13: 385-387

3 Wang X X, Tang X G, Chan H L W. Electromechanical and ferroelectric properties of $\left(\mathrm{Bi}_{1 / 2} \mathrm{Na}_{1 / 2}\right) \mathrm{TiO}_{3}-\left(\mathrm{Bi}_{1 / 2} \mathrm{~K}_{1 / 2}\right) \mathrm{TiO}_{3}-\mathrm{BaTiO}_{3}$ lead-free piezoelectric ceramics. Appl Phys Lett, 2004, 85: 91-94

4 Lin D M, Xiao D Q, Zhu J G, et al. Piezoelectric and ferroelectric properties of $\left[\mathrm{Bi}_{0.5}\left(\mathrm{Na}_{1-x-y} \mathrm{~K}_{x} \mathrm{Li}_{y}\right)_{0.5}\right] \mathrm{TiO}_{3}$ lead-free piezoelectric ceramics. Appl Phys Lett, 2006, 88: 062901-062904

5 Shout T R, Zhang S J. Lead-free piezoelectric ceramics: Alternatives for PZT? J Electroceram, 2004, 13: 385-388

6 Saito Y, Takao H, Tani T, et al. Lead-free piezoelectric ceramics. Nature, 2004, 84: 432-436

7 Du H L, Zhou W C, Luo F, et al. Design and electrical properties investigation of $\mathrm{K}_{0.5} \mathrm{Na}_{0.5} \mathrm{NbO}_{3}-\mathrm{BiMeO}_{3}$ lead-free piezoelectric ceramics. J Appl Phys, 2008, 104: 044104-044107

8 Du H L, Zhou W C, Luo F, et al. An approach to further improve piezoelectric properties of $\mathrm{K}_{0.5} \mathrm{Na}_{0.5}-\mathrm{NbO}_{3}$-based lead-free ceramics. Appl Phys Lett, 2007, 91: 202907-202909

$9 \mathrm{Du} \mathrm{H} \mathrm{L}$, Zhou W C, Luo F, et al. Sintering characteristic, microstructure, and dielectric relaxor behavior of $\left(\mathrm{K}_{0.5} \mathrm{Na}_{0.5}\right) \mathrm{NbO}_{3}-$ $\left(\mathrm{Bi}_{0.5} \mathrm{Na}_{0.5}\right) \mathrm{TiO}_{3}$ lead-free ceramics. J Am Ceram Soc, 2008, 91: 2903-2908

10 Du H L, Liu D J, Tang F S, et al. Microstructure, piezoelectric, and ferroelectric properties of $\mathrm{Bi}_{2} \mathrm{O}_{3}$-added $\left(\mathrm{K}_{0.5} \mathrm{Na}_{0.5}\right) \mathrm{NbO}_{3}$ lead-free ceramics. J Am Ceram Soc, 2007, 90: 2824-2827

11 Hollenstein E, Davis M, Damjanovic D, et al. Piezoelectric properties of Li- and Ta-modified $\mathrm{K}_{0.5} \mathrm{Na}_{0.5} \mathrm{NbO}_{3}$ ceramics. Appl Phys Lett, 2005, 87: 182905-182909

12 Zhang S J, Xia R, Shout T R, et al. Piezoelectric properties in perovskite $0.948\left(\mathrm{~K}_{0.5} \mathrm{Na}_{0.5}\right) \mathrm{NbO}_{3}-0.052 \mathrm{LiSbO}_{3}$ lead-free ceramics. J Appl Phys, 2006, 100:104108-104112

$13 \mathrm{Li} \mathrm{J} \mathrm{F}$, Wang K, Zhang B P, et al. Ferroelectric and piezoelectric properties of fine-grained $\mathrm{Na}_{0.5} \mathrm{~K}_{0.5} \mathrm{NbO}_{3}$ lead-free piezoelectric ceramics preparred by spark plasma sintering. J Am Ceram Soc, 2006, 89: 706-710

14 Yang Z P, Chang Y F, Wei L L. Phase transitional behavior and electrical properties of lead-free $\left(\mathrm{K}_{0.44} \mathrm{Na}_{0.52} \mathrm{Li}_{0.04}\right)\left(\mathrm{Nb}_{0.96-\mathrm{x}} \mathrm{Ta}_{\mathrm{x}} \mathrm{Sb}_{0.04}\right) \mathrm{O}_{3}$ piezoelectric ceramics. Appl Phys Lett, 2007, 90: 042911-042917

15 Guo Y, Kakimono K, Ohsata H. Phase transitional behavior and piezoelectric properties of $\left(\mathrm{Na}_{0.5} \mathrm{~K}_{0.5}\right) \mathrm{NbO}_{3}-\mathrm{LiNbO}_{3}$ ceramics. Appl Phys Lett, 2004, 85: 4121-4126

16 Zang G Z, Wang J F, Ghen H C, et al. Perovskite $\left(\mathrm{Na}_{0.5} \mathrm{~K}_{0.5}\right)_{1-x}(\mathrm{LiSb})_{x} \mathrm{Nb}_{1-x} \mathrm{O}_{3}$ lead-free piezoceramics. Appl Phys Lett, 2006, 88: 212908

17 Zuo R Z, Fang X S, Ye C. Structures and electrical properties of new lead free $\left(\mathrm{Na}_{0.5} \mathrm{~K}_{0.5}\right) \mathrm{NbO}_{3}-\left(\mathrm{Bi}_{0.5} \mathrm{Na}_{0.5}\right) \mathrm{TiO}_{3}$ ceramics. Appl Phys Lett, 2007, 90: 092904-092907

18 Hollenstein E, Damjanovic D, Setter N. Temperature stability of the piezoelectric properties of Li-modified KNN ceramics. J Eur Ceram Soc, 2007, 27: 4093-4098

19 Zhang S J, Xia R, Shout T R. Modified $\left(\mathrm{K}_{0.5} \mathrm{Na}_{0.5}\right) \mathrm{NbO}_{3}$ based lead-free piezoelectrics with broad temperature usage range. Appl Phys Lett, 2007, 91: 132913-132918

20 Wu J G, Xiao D Q, Wang Y Y, et al. CaTiO -modified $\left[\left(\mathrm{K}_{0.5} \mathrm{Na}_{0.5}\right)_{0.94} \mathrm{Li}_{0.06}\right]\left(\mathrm{Nb}_{0.94} \mathrm{Sb}_{0.06}\right) \mathrm{O}_{3}$ lead-free piezoelectric ceramics with improved temperature stability. Scrip Mater, 2008, 59: 750-755

21 Wu J G, Xiao D Q, Wang Y Y, et al. Improved temperature stability of $\mathrm{CaTiO}_{3}$-modified $\left[\left(\mathrm{K}_{0.5} \mathrm{Na}_{0.5}\right)_{0.96} \mathrm{Li}_{0.04}\right]\left(\mathrm{Nb}_{0.91} \mathrm{Sb}_{0.05} \mathrm{Ta}_{0.04}\right) \mathrm{O}_{3}$ lead-free piezoelectric ceramics. J Appl Phys, 2008, 104: 024102-024107

22 Du H L, Zhou W C, Luo F, et al. Perovskite lithium and bismuth modified potassium-sodium niobium lead-free ceramics for high temperature applications. Appl Phys Lett, 2007, 91: 182909-182912

23 Dai Y J, Zhang X W, Chen K P. Morphotropic phase boundary and electrical properties of $\mathrm{K}_{1-x} \mathrm{Na}_{x} \mathrm{NbO}_{3}$ lead-free ceramics. Appl Phys Lett, 2009, 94: 042905-042909

$24 \mathrm{Wu} \mathrm{L}$, Zhang J L, Wang C L, et al. Influence of compositional ratio K/Na on physical properties in $\mathrm{K}_{x} \mathrm{Na}_{1-x} \mathrm{NbO}_{3}$ ceramics. J Appl Phys, 2008, 103: 084116-084121

25 Cochran W. Crystal stability and the theory of ferroelectricity. Adv Phys, 1960, 9: 387-391 